\title{
Multi-objective optimization in optimal control problem with interval-valued objective function
}

\author{
Ulcilea A. Severino Leal ${ }^{*}, \quad$ Geraldo N. Silva ${ }^{\dagger}$, \\ *Universidade Federal de Mato Grosso do Sul, CPCS, \\ 79560-000, Chapadão do Sul, MS \\ †Depto de Matemática Aplicada, Universidade Estadual Paulista (UNESP) \\ 15054-000, São José do Rio Preto, SP \\ E-mail: ulcilea.leal@ufms.br, gsilva@ibilce.unesp.br,

\section{Weldon A. Lodwick} \\ UCDenver - Department of Mathematics \\ Denver, Colorado, USA \\ E-mail: Weldon.Lodwick@ucdenver.edu
}

\begin{abstract}
In this paper, we investigate an optimal control problem whose objective function has interval-valued. To minimization the interval-valued objective function, the concept of order relation on the interval space is considered. The order relations represent the decision maker's preference between two intervals and the optimal control problem is converted into a multiobjective control problem using the order relation. The maximize optimal control problem is also converted into a multi-objective problem using the order relation which represent the decision maker's preference between interval costs.
\end{abstract}

Keywords: Interval Analysis, Optimal Control, Multiple criteria problem

\section{Introduction}

The optimal control problems in general are assumed to have deterministic coefficients and fixed values. However, there are many situations where this assumption is not valid, and the problem involves some uncertainty. Thus, decision-making methods under uncertainty are required. The stochastic and the fuzzy programming were proposed to deal with this situation (see Bellman and Zadeh [3], Zimmerman [13]). Whereas the probability and the membership functions play important roles in each method, it is not always easy for the decision maker to specify them.

This paper proposes a simple decision making method under uncertainty apply for interval optimal control problems. The optimal control problem we are going to study is,

$$
\begin{aligned}
& \min I(x, u)=\int_{t_{0}}^{t_{1}} L(t, x(t), u(t)) d t \\
& \text { subject to: } u(t) \in U_{a d} \\
& x^{\prime}(t)=f(t, x(t), u(t)), \quad t \in\left(t_{0}, t_{1}\right) \\
& x\left(t_{0}\right)=x_{0},
\end{aligned}
$$

where $L(t, x(t), u(t))=[\underline{l}(t, x(t), u(t)), \bar{l}(t, x(t), u(t))], \underline{l}(t, x(t), u(t)) \leq \bar{l}(t, x(t), u(t))$ for all $t \in\left[t_{0}, t_{1}\right]$ is the interval-valued objective function, $f$ is a classical function, $x(t)$ is the state

\footnotetext{
${ }^{*} \dagger$ The research in this paper has been partially supported by both FAPESP-Brazil under the grants (2012/00189-3 and CEPID 2013/07375-0 of Industrial Mathematics) and CNPq under the grants (309335/20124 and 479109/2013 - 3) and Capes Foundation under the grant (11153/13-0).
} 
equation, $u(t)$ is the control function and $x_{0}$ is the initial condition of the problem. Moreover, $U_{a d}$ is the set of admissible controls functions and $X_{a d}$ is set admissible trajectory functions.

Ishibuchi and Tanaka [9] presented an approach to transform mathematical programming problems with interval-valued objective function into a multi-objective problem using the order relations that consider the left and right extremes, the center and the width of the interval-valued objective function.

The innovative result of this paper is the application of this concept to optimal control problems with interval-valued objective function. We convert the optimal control problem into a multi-objective control problem and define the optimal solution using the concept of Pareto optimality for multi-objective problems with interval order relation.

\section{Interval arithmetic}

This section describes the basic elements of the interval arithmetic and the generalized Hukuhara difference proposed in Stefanini [10] and used by Stefanini and Bede [11] to define the generalized Hukurara derivative for the case of interval-valued functions.

Let $\mathcal{K}_{C}^{n}$ be the space of nonempty compact and convex set of $\mathbb{R}^{n}$. If $n=1, \mathbb{I}(\mathbb{R})$ denotes the set of intervals of the real line, i.e., $\mathbb{I}(\mathbb{R})=\{[\underline{a}, \bar{a}] ; \underline{a}, \bar{a} \in \mathbb{R}$ and $\underline{a} \leq \bar{a}\}$, where $\underline{a}$ and $\bar{a}$ means the lower and upper bounds of any interval $A \in \mathbb{I}(\mathbb{R})$, respectively. Given two intervals $A=[\underline{a}, \bar{a}], B=[\underline{b}, \bar{b}] \in \mathbb{I}(\mathbb{R})$ and $k \in \mathbb{R}$ we consider the following operations

$$
\begin{aligned}
& A+B=[\underline{a}+\underline{b}, \bar{a}+\bar{b}], \\
& k A=\left\{\begin{array}{l}
{[k \underline{a}, k \bar{a}] \text { if } k \geq 0} \\
{[k \bar{a}, k \underline{a}] \text { if } k \leq 0,}
\end{array}\right.
\end{aligned}
$$

so that $A-B=A+(-1) B=[\underline{a}-\bar{b}, \bar{a}-\underline{b}]$. However, with these operations we do not have a linear space since there are no additive inverses, and therefore subtraction is not well-defined (see Aubin and Cellina [1]). Hukuhara [8] defined the Hukuhara difference (H-difference) for two intervals. This difference is unique, but it does not always exist. A generalization of the Hukuhara difference was introduced by Stefanini and Bede [11] in such a way that one always has a difference.

Definition 1. [11] The generalized Hukuhara difference (gH-difference, for short) of two intervals $A, B \in \mathbb{I}(\mathbb{R})$ is defined as follows

$$
A \ominus_{g} B=C,\left\{\begin{array}{l}
(a) \text { if } \quad A=B+C \text { or } \\
(b) \text { if } \quad B=A+(-1) C .
\end{array}\right.
$$

In case $(a)$, the gH-difference and H-difference are then equal. For gH-difference, since $A \ominus_{g} A=$ $[0,0]$, the gH-difference of the intervals $A=[\underline{a}, \bar{a}]$ and $B=[\underline{b}, \bar{b}]$ always exist, and it is equal to $A \ominus_{g} B=[\min \{\underline{a}-\underline{b}, \bar{a}-\bar{b}\}, \max \{\underline{a}-\underline{b}, \bar{a}-\bar{b}\}]$. An interval-valued function $F:\left[t_{0}, t_{1}\right] \rightarrow \mathbb{I}(\mathbb{R})$ is denoted by $F(t)=[\underline{f}(t), \bar{f}(t)]$ such that $\underline{f}(t) \leq \bar{f}(t)$ for all $t \in\left[t_{0}, t_{1}\right]$. The functions $\underline{f}(t)$ and $\bar{f}(t)$ will be called, respectively, the lower and upper functions of $F$.

\section{Multi-objective problem}

This section introduce the multi-objective problem. We consider problem of type (1) where the objective function is vector valued, i.e., $I: \mathbb{R}^{n} \times \mathbb{R}^{m} \rightarrow \mathbb{R}^{k}$, where $k$ is the number of objective functions. The solutions to the multi-objective problem are given with respect to the concept of dominance, where we will study three order relations $\leq$ in the multi-objective interval optimal control problem in view of these too definitions that follow. 
Definition 2. Let $X$ be any set and $f: \mathbb{R}^{n} \rightarrow \mathbb{R}^{k}$ a vector function. We say that a point $x^{*} \in X$ dominates the point $x \in X$ if $f\left(x^{*}\right) \leq f(x)$ and $f\left(x^{*}\right) \neq f(x)$, where $\leq$ is an order relation to be defined.

Definition 3. We say that $\left(x^{*}, u^{*}\right) \in X_{a d} \times U_{a d}$ is a Pareto optimal solution of the multi-objective control problem (minimization) if there exists no other solution $(x, u) \in X_{a d} \times U_{a d}$, such that $I(x, u) \leq I\left(x^{*}, u^{*}\right)$ and $I(x, u) \neq I\left(x^{*}, u^{*}\right)$. In other words, if $\left(x^{*}, u^{*}\right)$ is not dominated by any other feasible solution, where $X_{a d} \times U_{a d}$ is the admissible processes set solution.

There are many techniques for solving multi-objective optimization, some classic methods transform the multi-objective problem into a mono-objective problem. For example, the weight method, the $\epsilon$-restricted method, the weighted metrics method, the Benson method, and the target programming method, among others Haimes et al. [7] and Deb [6]. However, we will study the interval multi-objetive using the two above given definitions.

\section{Interval-valued optimal control problem}

This section presents a class of interval-valued optimal control problems and analyzes this problem from two different points of view. The optimal control problem mentioned in (1), where $f:\left[t_{0}, t_{1}\right] \times \mathbb{R}^{n} \times \mathbb{R}^{m} \rightarrow \mathbb{R}^{n}$ is a vector-valued function, $L: \mathbb{R} \times \mathbb{R}^{n} \times \mathbb{R}^{m} \rightarrow I(\mathbb{R})$ is the intervalvalued objective function, $x(t)$ is the state variable, $u(t)$ is the control variable, where $x(t)$ and $u(t)$ are related via the problem dynamics, $x^{\prime}(t)=f(t, x(t), u(t)), \quad t \in\left(t_{0}, t_{1}\right), x\left(t_{0}\right)=x_{0}$, and $u \in U_{a d}=C\left(\left[t_{0}, t_{1}\right] ; \mathbb{R}^{m}\right)$. For the problem described above the admissible trajectory set is give by

$$
X_{a d}:=\left\{x(t) \in C\left(\left[t_{0}, t_{1}\right] ; \mathbb{R}^{n}\right) ; x^{\prime}(t)=f(t, x(t), u(t)), u \in U_{a d}, \quad x\left(t_{0}\right)=x_{0}\right\} .
$$

The candidate solutions of the problem (1) are the admissible processes $(x(t), u(t)) \in X_{a d} \times U_{a d}$.

We denote by $L(t, x(t), u(t))=[\underline{l}(t, x(t), u(t)), \bar{l}(t, x(t), u(t))]$, with $\underline{l}(t, x(t), u(t)) \leq \bar{l}(t, x(t), u(t))$ for all $t \in\left[t_{0}, t_{1}\right]$. Using Aumann [2], we have that

$$
\int_{t_{0}}^{t_{1}} L(t, x(t), u(t)) d t=\left[\int_{t_{0}}^{t_{1}} \underline{l}(t, x(t), u(t)) d t, \int_{t_{0}}^{t_{1}} \bar{l}(t, x(t), u(t)) d t\right] .
$$

We will interpret a solution of problem (1) with a partial order relation in the space $\mathbb{I}(\mathbb{R})$. This is similar to the Pareto optimal solution concept used in multi-objective programming problems (see Ishibuchi and Tanaka [9], Wu [12] and Chalco-Cano et al. [4]). We study the problem using one order relation and compare to the other two order relations. First, we present the usual order relation, $\leq_{L U}$.

Let $A=[\underline{a}, \bar{a}]$ and $B=[\underline{b}, \bar{b}]$ be two intervals. The order relation $\leq_{L U}$ is defined as

$$
A \leq_{L U} B \text { if and only if } \underline{a} \leq \underline{b} \text { and } \bar{a} \leq \bar{b},
$$

and $A<_{L U} B$ if and only if $A \leq_{L U} B$ and $A \neq B$.

By center $a^{C}$ and the width $a^{S}$ we mean

$$
a^{C}=\frac{1}{2}(\bar{a}+\underline{a}) \text { and } a^{S}=(\bar{a}-\underline{a}) .
$$

The order relation $\leq_{L S}$ is defined by

1. for maximization

$$
A \geq_{L S} B \text { if and only if } \bar{a} \geq \bar{b} \text { and } a^{S} \leq b^{S},
$$

and $A>_{L S} B$ if and only if $A \geq_{L S} B$ and $A \neq B$. 
2. for minimization

$$
A \leq_{L S} B \text { if and only if } \underline{a} \leq \underline{b} \text { and } a^{S} \leq b^{S},
$$

and $A<_{L S} B$ if and only if $A \leq_{L S} B$ and $A \neq B$.

Let $\left(x^{*}(t), u^{*}(t)\right)$ be an admissible process of problem (1), i.e., $\left(x^{*}(t), u^{*}(t)\right) \in X_{a d} \times U_{a d}$. We say that $\left(x^{*}(t), u^{*}(t)\right)$ is an optimal LS-solution of problem (1) if there exists no $(x(t), u(t)) \in$ $X_{a d} \times U_{a d}$ such that $I(x(t), u(t))<_{L S} I\left(x^{*}(t), u^{*}(t)\right)$.

Ishibuchi and Tanaka [9] define a third order relation $\leq_{L C}$ by

1. for maximization

$$
A \leq_{L C} B \text { if and only if } \underline{a} \leq \underline{b} \text { and } a^{C} \leq b^{C},
$$

and $A<_{L C} B$ if and only if $A \leq_{L C} B$ and $A \neq B$.

2. for minimization

$$
A \leq_{U C} B \text { if and only if } \bar{a} \leq \bar{b} \text { and } a^{C} \leq b^{C},
$$

and $A<_{U C} B$ if and only if $A \leq_{U C} B$ and $A \neq B$.

Thus, analogously, if we let $\left(x^{*}(t), u^{*}(t)\right)$ be an admissible process of problem (1), i.e., $\left(x^{*}(t), u^{*}(t)\right) \in X_{a d} \times U_{a d}$, we say that $\left(x^{*}(t), u^{*}(t)\right)$ is an optimal UC-solution of problem (1) if there exists no $(x(t), u(t)) \in X_{a d} \times U_{a d}$ such that $I(x(t), u(t))<_{U C} I\left(x^{*}(t), u^{*}(t)\right)$.

Proposition 4.1. Let $A=[\underline{a}, \bar{a}]$ and $B=[\underline{b}, \bar{b}]$ be two intervals in $\mathbb{I}(\mathbb{R})$.

1. [9] If $A \leq_{L U} B$ then $A \leq_{U C} B$.

2. [4] If $A \leq_{L S} B$ then $A \leq_{L U} B$.

Proposition 4.2. Let $A=[\underline{a}, \bar{a}]$ and $B=[\underline{b}, \bar{b}]$ be two intervals in $\mathbb{I}(\mathbb{R})$. If $A \leq_{L S} B$ then $A \leq_{U C}$ $B$.

Proof. Follows from Proposition 4.1.

Theorem 4.1. Let $\left(x^{*}(t), u^{*}(t)\right)$ be an admissible process of problem (1), i.e., $\left(x^{*}(t), u^{*}(t)\right) \in$ $X_{a d} \times U_{a d}$. If $\left(x^{*}(t), u^{*}(t)\right)$ is an optimal UC-solution of problem (1) then it is an optimal $L S$-solution of problem (1).

Proof. It is easy to prove this by an indirect argument. Suppose that it is not the LS-solution. Then according to Proposition 4.2, you would find a contradiction.

A similar result is valid for the case of maximizing. In this case, we have that: let $\left(x^{*}(t), u^{*}(t)\right)$ be an admissible process of the maximization problem, i.e., $\left(x^{*}(t), u^{*}(t)\right) \in X_{a d} \times U_{a d}$. If $\left(x^{*}(t), u^{*}(t)\right)$ is an optimal LC-solution of the maximization problem then it is an optimal LSsolution for the maximization case.

\section{Formulation of the multi-objective control problem}

In this section, the optimal control problem with interval-valued objective function is formulated as a multi-objective control problem. Since the objective function $L(t, x(t), u(t))$ of problem (1) is an interval-valued function, the definition of optimal solution for this problem is a preference relation between intervals. Then, using only a of the order relations defined above and the concept of nondominated solutions defined in the previous section, we have the following results. 


\subsection{For minimization}

Consider (1). The optimal UC-solution of the problem can be obtained as the Pareto optimal solution of the following multi-objective control problem

$$
\begin{aligned}
& \min \left(\bar{I}(x, u), I^{C}(x, u)\right) \\
& \text { subject to: } u(t) \in U_{a d} \\
& x^{\prime}(t)=f(t, x(t), u(t)), \quad t \in\left(t_{0}, t_{1}\right) \\
& x\left(t_{0}\right)=x_{0},
\end{aligned}
$$

where $\bar{I}(x, u)$ and $I^{C}(x, u)$ are given by $\int_{t_{0}}^{t_{1}} \bar{l}(t, x(t), u(t)) d t$ and $\int_{t_{0}}^{t_{1}}\left(\frac{\bar{l}+\underline{l}}{2}\right)(t, x(t), u(t)) d t$, respectively. We have the following theorem.

Theorem 5.1. $\left(x^{*}, u^{*}\right)$ is an optimal UC-solution of problem (1) if and only if $\left(x^{*}, u^{*}\right)$ is a Pareto optimal solution of problem (7).

Proof. If $\left(x^{*}, u^{*}\right)$ is an optimal UC-solution, then there exists no $(x, u) \in X_{a d} \times U_{a d}$ such that $I(x, u) \leq_{U C} I\left(x^{*}, u^{*}\right)$ and $I(x, u) \neq I\left(x^{*}, u^{*}\right)$. Thus,

$$
\left\{\begin{array} { l } 
{ \overline { I } ( x , u ) \leq \overline { I } ( x ^ { * } , u ^ { * } ) } \\
{ \text { and } } \\
{ I ^ { C } ( x , u ) \leq I ^ { C } ( x ^ { * } , u ^ { * } ) }
\end{array} \quad \text { and } \left\{\begin{array}{l}
\bar{I}(x, u) \neq \bar{I}\left(x^{*}, u^{*}\right) \\
\text { and } \\
I^{C}(x, u) \neq I^{C}\left(x^{*}, u^{*}\right) .
\end{array}\right.\right.
$$

Therefore, $\left(x^{*}, u^{*}\right)$ is a nondominated solution, i.e., it is a Pareto optimal solution of problem (7).

Conversely, if $\left(x^{*}, u^{*}\right)$ is a Pareto optimal solution of problem $(7)$, i.e., $\left(x^{*}, u^{*}\right)$ is a nondominated solution, there exists no $(x, u) \in X_{a d} \times U_{a d}$ such that

$$
\begin{aligned}
& \left(\bar{I}(x, u), I^{C}(x, u)\right) \leq\left(\bar{I}\left(x^{*}, u^{*}\right), I^{C}\left(x^{*}, u^{*}\right)\right) \\
& \left(\bar{I}(x, u), I^{C}(x, u)\right) \neq\left(\bar{I}\left(x^{*}, u^{*}\right), I^{C}\left(x^{*}, u^{*}\right)\right) .
\end{aligned}
$$

This means that, there exists no $(x, u) \in X_{a d} \times U_{a d}$ such that $I(x, u)<_{U C} I\left(x^{*}, u^{*}\right)$. Therefore, $\left(x^{*}, u^{*}\right)$ is an optimal UC-solution of problem (1).

\subsection{For maximization}

We consider the following optimal control problem with interval-valued objective function

$$
\begin{aligned}
& \max I(x, u)=\int_{t_{0}}^{t_{1}} L(t, x(t), u(t)) d t \\
& \text { subject to: } u(t) \in U_{a d} \\
& x^{\prime}(t)=f(t, x(t), u(t)), \quad t \in\left(t_{0}, t_{1}\right) \\
& x\left(t_{0}\right)=x_{0} .
\end{aligned}
$$

The optimal LC-solution of problem (8) can be obtained as a Pareto optimal solutions of a multi-objective control problem, where the objective functions are $\underline{I}(x, u)$ and $I^{C}(x, u)$ given by $\int_{t_{0}}^{t_{1}} \underline{l}(t, x(t), u(t)) d t$ and $\int_{t_{0}}^{t_{1}}\left(\frac{\bar{l}+\underline{l}}{2}\right)(t, x(t), u(t)) d t$, respectively. For this case, we have the following theorem.

Theorem 5.2. $\left(x^{*}, u^{*}\right)$ is an optimal LC-solution of problem (8) if and only if $\left(x^{*}, u^{*}\right)$ is a Pareto optimal solution of the multi-objective control problem.

Proof. The proof is analogous to the proof of the previous theorem, considering the relation of order in the case of maximization. 


\section{Numerical example}

This section illustrates our proposed method for dealing with the interval-valued objective function. We consider the following linear quadratic optimal control problem with interval-valued objective function given by

$$
\begin{aligned}
& \max I(x, u)=-\frac{1}{2} \int_{0}^{1}(x(t)[0.9,1.1] x(t)+u(t)[0.9,1.1] u(t)) d t \\
& \text { subject to: } \\
& x^{\prime}(t)=u(t), \quad t \in(0,1) \\
& x(0)=1 .
\end{aligned}
$$

This problem is transformed into the following multi-objective control problem

$$
\begin{aligned}
& \max \left(\underline{I}(x, u), I^{C}(x, u)\right) \\
& \text { subject to: } \\
& x^{\prime}(t)=u(t), \quad t \in(0,1) \\
& x(0)=1 .
\end{aligned}
$$

Using some method to solve a multi-objective control problem (see Chankong and Haimes [5]), for we can obtain the Pareto optimal solution of problem (10). The technique utilized to resolve this problem was the weight method. A Pareto optimal solution of problem (10) is given below. In Figure (1a) the optimal control function solution of (10) is depicted. Figure (1b) depicts the optimal state function to (10). Lastly, Figure (2) depicts the optimal interval-valued objective function obtained as optimal control and state solutions. This solution is an optimal LC-solution of problem (9). It is also the optimal solution to the LS order problem (9).

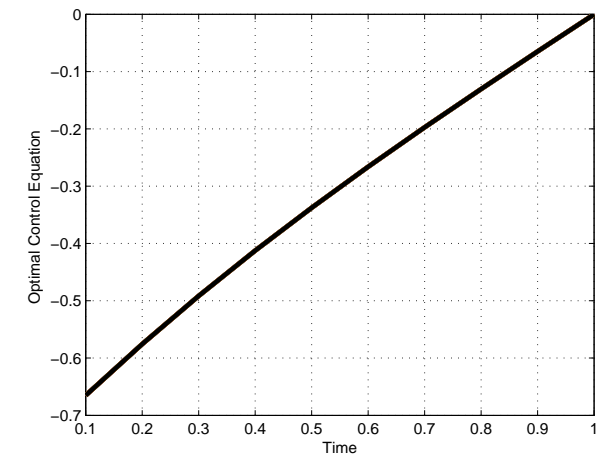

(a) Optimal control

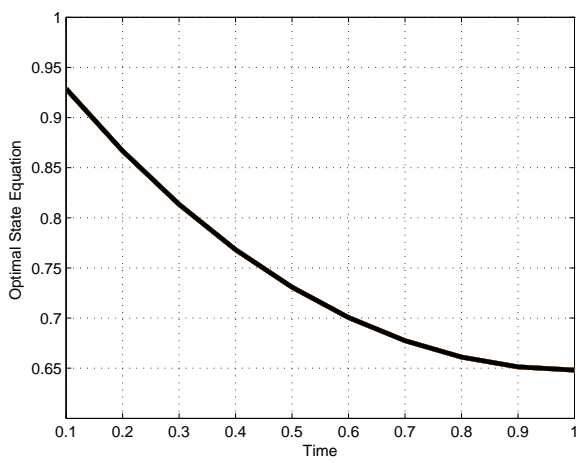

(b) Optimal state

Figure 1: Optimal process for the optimal control problem

\section{Conclusion}

In this paper, an optimal control problem with interval-valued objective function was converted into a multi-objective control problem, with two objectives for the minimization case: the right extreme and the center of the interval-valued objective function. The same occurs for the maximization problem. The solution sets of our optimal control problem with interval-valued objective function was defined as the Pareto optimal solutions of the corresponding multi-objective control problem. The proposed method was illustrated by a numerical example. 


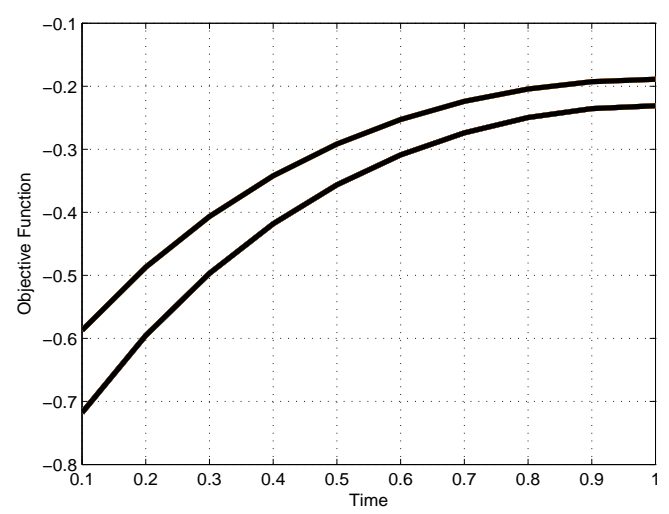

Figure 2: Interval-valued objective function

\section{References}

[1] J.P. Aubin and A. Cellina. Differential Inclusions. Springer-Verleg, New York, 1984.

[2] Robert J. Aumann. Integrals of set-valued functions. Journal of Mathematical Analysis and Applications, 12:1-12, 1965.

[3] R. E. Bellman and L. A. Zadeh. Decision-making in a fuzzy environment. Management Science, 17:141-164, 1970.

[4] Y. Chalco-Cano, A. Rufián-Lizana, H. Román-Flores, and M. D. Jiménez-Gamero. Calculus for interval-valued functions using generalized Hukuhara derivative and applications. Fuzzy Sets and Systems, 219:49-67, 2013.

[5] V. Chankong and Y. Y. Haimes. Multiobjective Decision Making: Theory and Methodology. North-Holland, 1983.

[6] K. Deb. Multi-Objevtive Optimization using Evolutionary Algorithms. John Wiley and Sons, New York, 2001.

[7] Y. Haimes, L. Lasdon, and D. Wismer. On a bicriterion formulation of the problems of integrated system identification and system optimization. IEEE Transactions on Systems, Man, and Cybernetics, 1(3):296-297, 1971.

[8] M. Hukuhara. Intégration des applications mesurables dont la valeur est un compact convex. Funkcial Ekvac, 10:205-229, 1967.

[9] Hisao Ishibuchi and Hideo Tanaka. Multiobjective programming in optimization of the interval objetive function. European Journal of Operation Research, 48:219-225, 1990.

[10] L. Stefanini. A generalizetion of Hukuhara difference for interval and fuzzy arithmetic. Series on Advances in Soft Computating, 48, 2008.

[11] L. Stefanini and B. Bede. Generalized Hukuhara differentiability of interval-valued functions and interval differential equations. Nonlinear Analysis, 71:1311-1328, 2009.

[12] H. C. Wu. The Karush-Kuhn-Tucker optimization conditions in an optimization problem with interval-valued objective function. European Journal of Operation Research, 176:4759, 2007.

[13] H. J. Zimmerman. Description and optimization of fuzzy systems. International Journal of General Systems, 2:206-215, 1976. 